\title{
A REMARK ON THE NONNEGATIVITY OF THE PANEITZ OPERATOR
}

\author{
MIJIA LAI
}

(Communicated by Guofang Wei)

\begin{abstract}
In this short article, we interpret the condition of a theorem of Gursky-Viaclovsky concerning the nonnegativity of the Paneitz operator as the metric being 3-positive Ricci. By a result of Wolfson, this condition can be preserved under the surgery of codimension $q \geq 3$. Combining these two observations, we expand the list of manifolds which admit metrics with a nonnegative Paneitz operator. Consequently, there exist metrics of constant $Q$-curvature on these manifolds.
\end{abstract}

\section{INTRODUCTION}

On a closed four manifold $\left(M^{4}, g\right)$, the Paneitz operator $P_{g}$ is defined as

$$
P_{g} u:=\Delta^{2} u-\delta\left\{\left(\frac{2}{3} R g-2 R i c\right) \circ \nabla u\right\},
$$

where $\delta: \Lambda^{1}\left(M^{4}\right) \rightarrow C^{\infty}\left(M^{4}\right)$ denotes the divergence operator. $P_{g}$ is a 4 -th order differential operator discovered by Paneitz in $1983[\mathrm{P}$. It is conformally invariant in the sense that under the conformal change of the metric $\tilde{g}=e^{2 w} g$, the Paneitz operator transforms as $P_{\tilde{g}}=e^{-4 w} P_{g}$.

The $Q$-curvature on $\left(M^{4}, g\right)$ is defined as

$$
Q=\frac{1}{12}\left(-\Delta R+R^{2}-3|R i c|^{2}\right) .
$$

Under the conformal change of the metric $\tilde{g}=e^{2 w} g, Q_{\tilde{g}}$ changes according to the following equation:

$$
P_{g} w+2 Q_{g}=2 Q_{\tilde{g}} e^{4 w}
$$

By integrating both sides of (1.3), we find the integral of the $Q$-curvature

$$
k_{g}:=\int_{M} Q_{g} d v_{g}
$$

is conformally invariant. Another point of view of the conformal invariance of $k_{g}$ is via the Chern-Gauss-Bonnet formula:

$$
4 \pi^{2} \chi(M)=\frac{1}{8} \int_{M}|W|^{2} d v_{g}+\int_{M} Q_{g} d v_{g},
$$

where $\chi(M)$ is the Euler characteristic of $M$ and $W$ is the Weyl tensor. Since $|W|^{2} d v_{g}$ is pointwise conformally invariant, it follows that the integral of the $Q$-curvature is also conformally invariant.

Received by the editors April 24, 2014 and, in revised form, July 15, 2014 and July 31, 2014. 2010 Mathematics Subject Classification. Primary 53A30. 
The pair $(P, Q)$ is analogous to the pair $(\Delta, K)$, the Laplace operator and the Guassian curvature in dimension two, where

$$
\Delta u+K_{\tilde{g}} e^{2 u}=K_{g}, \quad \text { for } \tilde{g}=e^{2 u} g .
$$

Upon integrating both sides, one finds the connection with the Gauss-Bonnet formula:

$$
\int_{M} K_{\tilde{g}} e^{2 u} d v_{g}=\int_{M} K_{\tilde{g}} d v_{\tilde{g}}=\int_{M} K_{g} d v_{g}=2 \pi \chi(M) .
$$

Another important curvature in conformal geometry is the Schouten tensor $A=$ Ric $-\frac{R}{2(n-1)} g$. The Riemann curvature tensor has a nice decomposition:

$$
R m=W \oplus \frac{1}{n-2} A \odot g,
$$

where $\odot$ stands for the Kulkarni-Nomizu product. Owing to this decomposition and conformal invariance of the Weyl tensor, it suffices to understand the conformal change of the Schouten tensor in order to understand the conformal change of the full Riemann curvature tensor.

Let $\sigma_{2}(A)$ denote the second elementary symmetric polynomial applied to the eigenvalues of the Schouten tensor with respect to the Riemannian metric. In dimension four, we have

$$
\sigma_{2}(A)=\frac{1}{6} R^{2}-\frac{1}{2}|R i c|^{2},
$$

thus the conformal invariant $k_{g}$ can also be expressed as

$$
k_{g}=\frac{1}{2} \int_{M} \sigma_{2}(A) d v_{g}
$$

The $Q$-curvature and the Paneitz operator play important roles in studying the geometry of four-manifolds and appear in diverse topics (see e.g. BCY, CGY, ES, FG, GZ]). One problem which has attracted a great deal of interest in the past two decades is whether or not one can find a metric of constant $Q$-curvature on a given compact Riemannian manifold. The first breakthrough is due to Chang-Yang [CY] by a variational method.

Consider the functional

$$
F(u)=\left\langle P_{g} u, u\right\rangle+4 \int_{M} Q_{g} u d v_{g}-k_{g} \log \int_{M} e^{4 u} d v_{g} .
$$

Its Euler-Lagrange equation is

$$
P_{g} u+2 Q_{g}=2 \overline{Q_{g}} e^{4 u} ; \quad \overline{Q_{g}} \text { a constant. }
$$

Hence a metric $\tilde{g}=e^{2 u} g$ with constant $Q$-curvature can be found by seeking critical points of (1.5).

Theorem 1.1 (Chang-Yang). Let $(M, g)$ be a compact four-manifold without boundary. Assume (1) the Paneitz operator $P_{g}$ is nonnegative with $\operatorname{Ker} P_{g}=$ $\left\{\right.$ constants\}, and (2) the conformal invariant $k_{g}<8 \pi^{2}$. Then there exists a global minimizer of $F$, which satisfies (1.6). Note here $8 \pi^{2}$ is the value of $k_{g}$ for the round sphere.

More recently, Djadli and Malchiodi $[\mathrm{DM}]$ proved

Theorem 1.2 (Djadli-Malchiodi). Suppose $\operatorname{Ker} P_{g}=\{$ constants $\}$, and assume that $k_{g} \neq 8 k \pi^{2}$ for $k=1,2, \cdots$. Then $(M, g)$ admits a conformal metric with constant $Q$-curvature. 
In view of the above theorems, the spectral properties of the Paneitz operator are closely related to the existence problem of metrics with constant $Q$-curvature. In this regard, Gursky G2 proved nonnegativity of the Paneitz operator under natural assumptions on two conformal invariants; one is the above-mentioned $k_{g}$, the other one is the Yamabe constant:

$$
Y(g)=\inf _{\tilde{g}=e^{2 u}} \frac{\int R_{\tilde{g}} d v_{\tilde{g}}}{\operatorname{vol}(\tilde{g})^{1 / 2}} .
$$

Theorem 1.3 (Gursky). Suppose $\left(M^{4}, g\right)$ has both $Y(g) \geq 0$ and $k_{g} \geq 0$. Then the Paneitz operator is nonnegative, and $\operatorname{Ker}\left(P_{g}\right)=\{$ constants $\}$.

On the other hand, Eastwood and Singer ES have constructed metrics on $k\left(S^{3} \times S^{1}\right)$ for all $k>0$ with $P_{g} \geq 0$ and $\operatorname{Ker}\left(P_{g}\right)=\{$ constants $\}$. Yet these manifolds have $k_{g}<0$. Hence a more general condition to imply the nonnegativeness of the Paneitz operator is desirable. Indeed, Gurksy-Viaclovsky [GV] proved the nonnegativity of the Paneitz operator on $\left(M^{4}, g\right)$ with positive scalar curvature under the weaker assumption

$$
k_{g}+\frac{1}{6} Y(g)^{2}>0
$$

In this article, we make some remarks on the nonnegativity of the Paneitz operator in the spirit of above-mentioned results.

Following $[\mathrm{W},(M, g)$ is said to have $k$-positive Ricci curvature if the sum of the $k$ smallest eigenvalues of Ricci curvature is positive everywhere on $M$.

Theorem 1.4. Suppose $\left(M^{4}, g\right)$ has 3-positive Ricci curvature. Then the Paneitz operator $P_{g}$ is nonnegative, and $\operatorname{Ker}\left(P_{g}\right)=\{$ constants $\}$.

Remark 1.5. This theorem is merely a reformulation of Proposition 6.1 in [GV].

One should point out that the condition 3-positive Ricci is not conformally invariant. We know the scalar curvature $\tilde{R}$, under the conformal change of $\tilde{g}=\varphi^{2} g$, changes as

$$
\tilde{R}=\varphi^{-3}\left(-6 \Delta_{g} \varphi+R_{g} \varphi\right) .
$$

Choose $\varphi=u_{1}-\min u_{1}+\epsilon>0$, where $u_{1}$ is the eigenfunction corresponding to the first eigenvalue of $\lambda_{1}$ of $\Delta_{g}$, i.e., $\Delta u_{1}=-\lambda_{1} u_{1}$. Clearly, $u_{1}$ changes sign, and hence $\min u_{1}<0$. With this choice of $\varphi$, we find that

$$
\tilde{R}=\varphi^{-3}\left(6 \lambda_{1} u_{1}+R_{g} \varphi\right)
$$

can be made negative at the point where $u_{1}$ attains its minimum by choosing $\epsilon$ small enough. Hence the resulting conformal metric $\tilde{g}=\varphi^{2} g$ is not 3-positive Ricci as its scalar curvature is negative somewhere.

Wolfson showed in W] that for four-manifolds, 3-positive Ricci curvature can be preserved under surgeries of codimension $q \geq 3$. Here we briefly recall the definition of performing a surgery of codimension $q$ on $M^{n}$. The starting point is the following relation:

$$
\partial\left(S^{p} \times D^{q}\right)=S^{p} \times S^{q-1}=\partial\left(D^{p+1} \times S^{q-1}\right) .
$$

Given an embedding $S^{n-q} \times D^{q} \subset M$, let

$$
M^{\prime}=M \backslash\left(S^{n-q} \times D^{q}\right) \cup_{S^{n-q} \times S^{q-1}}\left(D^{n-q+1} \times S^{q-1}\right) .
$$

That is, $M^{\prime}$ is obtained from $M$ by removing an embedded $S^{n-q} \times D^{q}$ and replacing it with $D^{n-q+1} \times S^{q-1}$, glued along the common boundary. We say $M^{\prime}$ is obtained 
from $M$ by performing a surgery of codimension $q$. Taking connected sum can be viewed as a special surgery of codimension $n$ on manifolds with two connected components.

A direct consequence of Wolfson's result is

Corollary 1.6. Let $M$ be a compact four-manifold with a metric of 3-positive Ricci curvature. Then any manifold obtained from $M$ by performing surgeries of codimension $q \geq 3$ also admits a metric of 3-positive Ricci curvature, and thus the associated Paneitz operator is nonnegative with trivial kernel. In particular, if $M_{1}$ and $M_{2}$ are manifolds with 3-positive Ricci curvature, then the manifold

$$
N=k M_{1} \# l M_{2}, \quad k, l \in \mathbb{N}
$$

admits a metric with respect to which the Paneitz operator is nonnegative and with trivial kernel.

Remark 1.7. Some obvious choices of $M_{1}$ and $M_{2}$ are: $S^{4}, S^{3} \times S^{1}, S^{2} \times S^{2}, S^{2} \times T^{2}$, $\mathbb{C P}^{2}, \overline{\mathbb{C P}}^{2}, \Sigma_{g} \times S_{r}^{2}$ and their quotients. In the last one, $\Sigma_{g}$ is a Riemann surface of genus $g$ endowed with the hyperbolic metric and $S_{r}^{2}$ is the round sphere with sufficiently small radius $r$. This list expands the known examples of manifolds which admit metrics with a nonnegative Paneitz operator. It also provides examples complementary to the condition (1.7).

Example. Let $M=\Sigma_{g} \times S_{\frac{1}{2}}^{2}$ with the product metric. The Ricci curvature has eigenvalues $(-1,-1,4,4)$ which is of 3 -positive Ricci curvature. $A_{g}=R i c-\frac{R}{6} g$ has eigenvalues $(-2,-2,3,3)$. A simple calculation shows

$$
k_{g}=\frac{1}{2} \int_{M} \sigma_{2}\left(A_{g}\right)=-22(g-1) \pi^{2} .
$$

By a classical result of Aubin, $Y(g)<Y\left(S^{4}\right)$. Hence condition (1.7) fails to hold on $M$ when $g$ is sufficiently large. By taking connected sum, one can produce additional examples complementary to the condition (1.7).

Remark 1.8. In fact Theorem 1.4 holds under the weaker condition that the metric is of 3-nonnegative Ricci curvature (cf. Proposition 6.1 of [GV]). It is unclear if this condition can be preserved under performing surgeries. Nevertheless, one cannot generally conformally deform it to be a metric of 3-positive Ricci. $T^{4}$ serves as a simple example. The flat metric is 3 -nonnegative Ricci. However, were there a metric of 3-positive Ricci, it contradicts the well-known theorems of Schoen-Yau and Gromov-Lawson [SY, GL] on the nonexistence of metrics with positive scalar curvature on $T^{n}$.

Finally, since 3-positive Ricci curvature implies positive scalar curvature, on such manifolds we necessarily have $Y(g)>0$. In addition, Gursky showed [G2] that if $Y(g) \geq 0$, then $k_{g} \leq 8 \pi^{2}$ with equality holding if and only if $M$ is conformally equivalent to the round sphere. In view of this characterization, combining Corollary 1.6 and Theorem [1.1, we obtain

Theorem 1.9. Let $M$ be a closed four-manifold with 3-positive Ricci curvature. Then any manifold obtained from $M$ by performing surgeries of codimension $q \geq 3$ admits metrics of constant $Q$-curvature. 


\section{ProOfS}

For the reader's convenience and the following propositions, we include a proof of Theorem 1.4.

Proof of Theorem 1.4. Let $\varphi \in C^{\infty}(M)$ be any function. We wish to estimate

$$
\int_{M} \varphi P_{g} \varphi=\int_{M}(\Delta \varphi)^{2}+\int_{M} \frac{2}{3} R|\nabla \varphi|^{2}-2 \operatorname{Ric}(\nabla \varphi, \nabla \varphi) .
$$

By Newton-Maclaurin's inequality $\frac{\sigma_{1}}{n} \geq\left(\frac{\sigma_{2}}{\left(\begin{array}{c}n \\ 2\end{array}\right)}\right)^{\frac{1}{2}}$, we have

$$
(\Delta u)^{2}=\left(\sigma_{1}\left(D^{2} u\right)\right)^{2} \geq \frac{8}{3} \sigma_{2}\left(D^{2} u\right) .
$$

By Reilly's formula $[\underline{\mathrm{R}}$ on compact closed manifold,

$$
\int_{M} 2 \sigma_{2}\left(D^{2} u\right)=\int_{M} \operatorname{Ric}(\nabla u, \nabla u)
$$

(2.1) becomes

$$
\int_{M} \varphi P_{g} \varphi \geq \frac{2}{3}\left[\int_{M} R|\nabla \varphi|^{2}-\operatorname{Ric}(\nabla \varphi, \nabla \varphi)\right] \geq \frac{2}{3} \int_{M}\left(\lambda_{1}+\lambda_{2}+\lambda_{3}\right)|\nabla \varphi|^{2} \geq 0 .
$$

Here we denote the three smallest eigenvalues of Ricci curvature by $\lambda_{1}, \lambda_{2}, \lambda_{3}$. Since $g$ is 3-positive Ricci curvature, we get the desired inequality. Moreover, the equality holds if and only if $|\nabla \varphi|=0$, and thus $\operatorname{Ker}\left(P_{g}\right)=\{$ constants $\}$.

Remark 2.1. This computation also provides an alternative proof of Theorem 1.3 in the case $Y(g)>0$ and $k_{g}>0$. Under these assumptions, by [CGY], there exists a metric $\tilde{g}$ conformal to $g$ with $\sigma_{2}\left(A_{\tilde{g}}\right)>0$. The positivity of $\sigma_{2}\left(A_{\tilde{g}}\right)$ implies the scalar curvature cannot change the sign. Since $Y(g)>0, R_{\tilde{g}}$ is positive everywhere on $M$. It follows (see [CGY] that Ricci curvature is positively pinched:

$$
\left(\frac{1}{2} R_{\tilde{g}}-\frac{3 \sigma_{2}\left(A_{\tilde{g}}\right)}{R_{\tilde{g}}}\right) \tilde{g} \geq \operatorname{Ric}_{\tilde{g}} \geq \frac{3 \sigma_{2}\left(A_{\tilde{g}}\right)}{R_{\tilde{g}}} \tilde{g}>0 .
$$

Therefore, the nonnegativity of the Paneitz operator follows.

As an aside, we prove a lower bound on the first positive eigenvalue of the Paneitz operator.

Proposition 2.2. Let $\left(M^{4}, g\right)$ be a closed Riemannian manifold. Suppose $Y(g)>0$ and $\sigma_{2}\left(A_{g}\right) \geq \kappa>0$. Denote the first positive eigenvalue of $\Delta_{g}$ by $\lambda_{1}$. Let $\mu_{1}$ be the first positive eigenvalue of $P_{g}$; then

$$
\mu_{1} \geq \frac{4}{3} \sqrt{3 \kappa} \lambda_{1}
$$

Proof. By Rayleigh's quotients characterization,

$$
\mu_{1}=\inf _{\int_{M} \varphi=0} \frac{\int_{M} \varphi P_{g} \varphi}{\int_{M} \varphi^{2}} .
$$


Let $\varphi \neq 0$ satify $\int_{M} \varphi=0$. Then we proceed from (2.2) as follows:

$$
\begin{aligned}
\int_{M} \varphi P_{g} \varphi & \geq \frac{2}{3}\left(\int_{M} R|\nabla \varphi|^{2}-\operatorname{Ric}(\nabla \varphi, \nabla \varphi)\right) \\
& =\frac{2}{3}\left(\int_{M} \frac{3}{4} R|\nabla \varphi|^{2}-E(\nabla \varphi, \nabla \varphi)\right) \\
& \geq \frac{2}{3} \int_{M}\left(\frac{3}{4} R-\frac{\sqrt{3}}{2}|E|\right)|\nabla \varphi|^{2} .
\end{aligned}
$$

Here $E(\cdot, \cdot)$ is the traceless part of Ricci curvature, and we have used the sharp inequality

$$
|E(\nabla \varphi, \nabla \varphi)| \leq \frac{\sqrt{3}}{2}|E||\nabla \varphi|^{2} .
$$

By the previous remark, if $Y(g)>0$ and $\sigma_{2}\left(A_{g}\right)>0$, then $R$ is positive everywhere on $M$. Notice that

$$
\sigma_{2}\left(A_{g}\right)=\frac{1}{24} R^{2}-\frac{1}{2}|E|^{2} \geq \kappa>0 .
$$

Then a Lagrange multiply method leads to

$$
\frac{3}{4} R-\frac{\sqrt{3}}{2}|E| \geq 2 \sqrt{3 \kappa} \text {. }
$$

It then follows that

$$
\begin{aligned}
\int_{M} \varphi P_{g} \varphi & \geq \frac{2}{3} \int_{M}\left(\frac{3}{4} R-\frac{\sqrt{3}}{2}|E|\right)|\nabla \varphi|^{2} \\
& \geq \frac{4}{3} \sqrt{3 \kappa} \int_{M}|\nabla \varphi|^{2} \geq \frac{4}{3} \sqrt{3 \kappa} \lambda_{1} \int_{M} \varphi^{2} .
\end{aligned}
$$

A slight modification of the above calculation applies to four-manifolds with boundary.

Proposition 2.3. Let $\left(M^{4}, g\right)$ be a compact four-manifold with boundary. Assume $Y(M, \partial M, g)>0$ and $\sigma_{2}(A) \geq \kappa>0$. Denote the first eigenvalue of $\Delta_{g}$ with Dirichlet boundary condition by $\lambda_{1}$ and denote the first eigenvalue of $\Delta_{g}$ with Neumann boundary condition by $\nu_{1}$. Let $\mu_{1}$ be the first positive eigenvalue of $P_{g}$; then

$$
\mu_{1} \geq \frac{4}{3} \sqrt{3 \kappa} \max \left\{\lambda_{1}, \nu_{1}\right\} .
$$

Proof. Let $u$ be the solution to the first eigenvalue problem

$$
\begin{cases}P_{g} u=\mu_{1} u & \text { in } M \\ u=\frac{\partial u}{\partial n}=0 & \text { on } \partial M\end{cases}
$$

Then

$$
\begin{aligned}
\mu_{1} \int_{M} u^{2} & =\int_{M} u P_{g} u \\
& =\int_{M}(\Delta u)^{2}+\int_{M} \frac{2}{3} R|\nabla u|^{2}-2 \operatorname{Ric}(\nabla u, \nabla u) \\
& \geq \frac{8}{3} \int_{M} \sigma_{2}\left(D^{2} u\right)+\int_{M} \frac{2}{3} R|\nabla u|^{2}-2 \operatorname{Ric}(\nabla u, \nabla u) .
\end{aligned}
$$


Reilly's formula $[\mathrm{R}]$ for manifolds with boundary reads

$$
\int_{M} 2 \sigma_{2}\left(D^{2} u\right)=\int_{\partial M}(\Delta z-f H) f-\nabla z \cdot \nabla f-\Pi(\nabla z, \nabla z)+\int_{M} \operatorname{Ric}(\nabla u, \nabla u),
$$

where $z=\left.u\right|_{\partial M}, f=\frac{\partial u}{\partial n}, H$ is the mean curvature of the boundary and $\Pi$ is the second fundamental form. In view of the boundary conditions on $u$, the above formula reduces to

$$
\int_{M} 2 \sigma_{2}\left(D^{2} u\right)=\int_{M} \operatorname{Ric}(\nabla u, \nabla u)
$$

Now the calculation in Proposition 2.2 carries over verbatim. The desired inequality follows since $u$ satisfies both the Dirichlet boundary condition and the Neumann boundary condition.

Remark 2.4. Estimates in the same spirit were obtained in $[\mathrm{XY}]$, where they also proved an interesting result that the positivity of the Paneitz operator is preserved in dimension greater than four by taking connected sum.

\section{ACKNOWLEDGEMENTS}

The author would like to thank Hao Fang for helpful conversations, and the referee, whose comments and suggestions improved the final version of this article.

\section{REFERENCES}

[BCY] Thomas P. Branson, Sun-Yung A. Chang, and Paul C. Yang, Estimates and extremals for zeta function determinants on four-manifolds, Comm. Math. Phys. 149 (1992), no. 2, 241-262. MR 1186028 (93m:58116)

[CGY] Sun-Yung A. Chang, Matthew J. Gursky, and Paul C. Yang, An equation of MongeAmpère type in conformal geometry, and four-manifolds of positive Ricci curvature, Ann. of Math. (2) 155 (2002), no. 3, 709-787, DOI 10.2307/3062131. MR1923964|(2003j:53048)

[CY] Sun-Yung A. Chang and Paul C. Yang, Extremal metrics of zeta function determinants on 4-manifolds, Ann. of Math. (2) 142 (1995), no. 1, 171-212, DOI 10.2307/2118613. MR 1338677 (96e:58034)

[DM] Zindine Djadli and Andrea Malchiodi, Existence of conformal metrics with constant Qcurvature, Ann. of Math. (2) 168 (2008), no. 3, 813-858, DOI 10.4007/annals.2008.168.813. MR2456884(2009h:53074)

[ES] Michael G. Eastwood and Michael A. Singer, The Fröhlicher [Frölicher] spectral sequence on a twistor space, J. Differential Geom. 38 (1993), no. 3, 653-669. MR.1243789 (94k:32050)

[FG] Charles Fefferman and C. Robin Graham, Q-curvature and Poincaré metrics, Math. Res. Lett. 9 (2002), no. 2-3, 139-151, DOI 10.4310/MRL.2002.v9.n2.a2. MR.1909634 (2003f:53053)

[G1] Matthew J. Gursky, The Weyl functional, de Rham cohomology, and Kähler-Einstein metrics, Ann. of Math. (2) 148 (1998), no. 1, 315-337, DOI 10.2307/120996. MR.1652920 (99i:58036)

[G2] Matthew J. Gursky, The principal eigenvalue of a conformally invariant differential operator, with an application to semilinear elliptic PDE, Comm. Math. Phys. 207 (1999), no. 1, 131-143, DOI 10.1007/s002200050721. MR.1724863 (2000k:58029)

[GL] Mikhael Gromov and H. Blaine Lawson Jr., The classification of simply connected manifolds of positive scalar curvature, Ann. of Math. (2) 111 (1980), no. 3, 423-434, DOI 10.2307/1971103. MR.577131 (81h:53036)

[GV] Matthew J. Gursky and Jeff A. Viaclovsky, A fully nonlinear equation on four-manifolds with positive scalar curvature, J. Differential Geom. 63 (2003), no. 1, 131-154. MR.2015262 (2004h:53052) 
[GZ] C. Robin Graham and Maciej Zworski, Scattering matrix in conformal geometry, Invent. Math. 152 (2003), no. 1, 89-118, DOI 10.1007/s00222-002-0268-1. MR.1965361 (2004c:58064)

[P] Stephen M. Paneitz, A quartic conformally covariant differential operator for arbitrary pseudo-Riemannian manifolds (summary), SIGMA Symmetry Integrability Geom. Methods Appl. 4 (2008), Paper 036, 3, DOI 10.3842/SIGMA.2008.036. MR.2393291 (2009b:58080)

[R] Robert C. Reilly, Applications of the Hessian operator in a Riemannian manifold, Indiana Univ. Math. J. 26 (1977), no. 3, 459-472. MR0474149 (57 \#13799)

[SY] R. Schoen and S. T. Yau, On the structure of manifolds with positive scalar curvature, Manuscripta Math. 28 (1979), no. 1-3, 159-183, DOI 10.1007/BF01647970. MR535700 (80k:53064)

[W] Jon Wolfson, Manifolds with k-positive Ricci curvature, Variational problems in differential geometry, London Math. Soc. Lecture Note Ser., vol. 394, Cambridge Univ. Press, Cambridge, 2012, pp. 182-201. MR2882775 (2012k:53070)

[XY] Xingwang Xu and Paul C. Yang, Positivity of Paneitz operators, Discrete Contin. Dynam. Systems 7 (2001), no. 2, 329-342, DOI 10.3934/dcds.2001.7.329. MR.1808405 (2002d:58043)

Department of Mathematics, Shanghai Jiaotong University, 800 Dongchuan Road, Shanghai 200240, People's Republic of China

E-mail address: laimijia@sjtu.edu.cn 